\title{
Increased expression of RRM2 by human papillomavirus E7 oncoprotein promotes angiogenesis in cervical cancer
}

\author{
$\mathrm{N}$ Wang ${ }^{1}$, T Zhan ${ }^{1}, \mathrm{~T} \mathrm{Ke}^{1}, \mathrm{X} \mathrm{Huang}^{2}$, D Ke ${ }^{3}$, Q Wang ${ }^{\star, 1,4}$ and $\mathrm{H} \mathrm{Li}{ }^{\star, 1}$ \\ ${ }^{1}$ Key Laboratory of Molecular Biophysics of the Ministry of Education, College of Life Science and Technology, Center for Human \\ Genome Research, Cardio-X Institute, Huazhong University of Science and Technology, Wuhan 430074, China; ${ }^{2}$ Division \\ of Medical Oncology, Puai District of Huangshi Central Hospital, Wuhan, China; ${ }^{3}$ Division of Bone, Daye Hospital of Traditional \\ Chinese Medicine, Wuhan, China and ${ }^{4}$ Center for Cardiovascular Genetics, Department of Molecular Cardiology, Lerner Research \\ Institute, Cleveland Clinic, Cleveland, OH, USA
}

Background: The purpose of this study was to confirm that RRM2 as a novel target of HPVE7 involved in cervical cancer angiogenesis.

Methods: Gene expression was analysed by RT-gPCR, western blot and immunohistochemistry in cervical cancer tissue and cell lines. Luciferase reporter assay was used to determine the activities of various RRM2 promoters. Secreted VEGF was measured by ELISA. RRM2-mediated capillary tube formation induced by HPVE7 in cervical cancer cells were evaluated using human umbilical vein endothelial cells in vitro. ROS induced by RRM2 in cercal cancer cells was confirmed by flow cytometry. The growth of cervical cancer cell overexpression RRM2 was examined by nude mouse xenograft.

Results: RRM2 as a novel downstream target for HPVE7 was upregulated by it at the transcriptional level through the E7-pRb interaction and binding of E2F to the RRM2 promoter region. Immunohistochemical analysis showed that the level of RRM2 positively correlated with the HPVE7 level in human cervical cancer. Functionally, overexpression of RRM2 enhanced the expression of HIF-1 $\alpha$ and VEGF via activation of the ERK1/2 signalling pathway in cervical cancer cells, and significantly associated with increased microvessel densities in cervical cancer tissues. In vitro, HPVE7 stimulated RRM2-dependent capillary tube formation by HUVECs, and RRM2-enhanced angiogenesis was VEGF dependent. RRM2-activated ERK1/2 pathway was mediated through production of ROS. In the xenograft mouse model, overexpression of RRM2 in cervical cancer cells enhanced tumour growth as well as microvessel densities.

Conclusion: HPVE7 induces upregulation of RRM2, which then promotes cervical carcinogenesis via ROS-ERK1/2-HIF-1 $\alpha$-VEGFinduced angiogenesis. Thus, the inhibition of RRM2 activity may be a novel therapeutic strategy for human cervical cancer.

Cervical cancer is the second leading cause of cancer-related mortalities in women worldwide (Jemal et al, 2011). Epidemiological studies have shown that persistent infection by specific types of human papilloma viruses (HPVs), such as type 16 and 18 , is a causal agent for human cervical cancer (Bosch and de, 2007). The transforming activity of HPVs is dependent on functions of two viral oncoproteins, E6 and E7, both of which are consistently expressed in cervical tumours. The E6 protein leads to the rapid ubiquitination and degradation of p53 tumour suppressor, which consequently deregulates the p53 transcriptional pathway (Werness et al, 1990). In contrast, the E7 protein targets and inactivates the retinoblastoma protein $(\mathrm{Rb})$, resulting in the release of active $\mathrm{E} 2 \mathrm{~F}$ and the activation of E2F target genes (Huh et al, 2007). Although disruption of the p53 and pRb pathways by the E6 and E7 proteins,

*Correspondence: Dr H Li; E-mail: huili0930@mail.hust.edu.cn or Dr Q Wang; E-mail: qkwang@mail.hust.edu.cn

Received 28 September 2013; revised 4 December 2013; accepted 10 December 2013; published online 14 January 2014

(c) 2014 Cancer Research UK. All rights reserved 0007-0920/14 
respectively, contributes to the transforming capacity of HPVs, the precise regulatory pathways downstream of these two tumour suppressors remain elusive in cervical cancer. To identify novel potential downstream genes for HPVE7, we analysed four publicly available microarray data sets (Garner-Hamrick et al, 2004; Thierry et al, 2004; Rosty et al, 2005; Kuner et al, 2007) and detected a set of overlap genes whose mRNA expression upregulated at least twofold in cervical cancer tissue and cell lines in these data sets. Then we searched the DNA sequence upstream of these genes for the core binding sequence for E2F with TFSEARCH (ver.1.3) program. RRM2, encoding the ribonucleotide reductase small subunit M2, is one of the above genes shown to be significantly upregulated and contains two putative E2F-binding sites within its promoter region, which suggested that RRM2 might be a potential target activated by the HPVE7. The RRM2 protein is a component of ribonucleotide reductase (RNR), a key enzyme that reduces ribonucleoside diphosphate (NDP) to deoxyribonucleoside diphosphate (dNDP), and is required for DNA synthesis (Nordlund and Reichard, 2006). It is well known that RNR activity is positively correlated with cancer cell division (Elford et al, 1970). A substantial body of evidences demonstrated that RRM2 was tightly linked to the biological behaviour of cancers. For example, overexpression of RRM2 correlated with cellular invasiveness, metastasis, tumour angiogenesis and poor patient outcome (Zhou et al, 1998; Zhang et al, 2009; Liu et al, 2013). However, the malignant phenotypes caused by a high level of RRM2 expression might be independent of RNR activity for the following reason. Similar to RRM2, overexpression of RRM1 (also a subunit of RNR) resulted in enhanced RNR activity and increased dNDP synthesis. However, in contrast to overexpression of RRM2, overexpression of RRM1 inhibited the invasive and metastatic potential of tumours (Gautam et al, 2003). Thus, there was no correlation between the level of RNR activity and tumour malignant phenotypes, and RRM2 may have a critical role in tumour progression beyond its capacity of dNDP production.

The characteristics of RRM2 to promote tumour progression are tightly associated with its capability to induce activities of a variety of oncogenes, including those encoding NF- $\kappa \mathrm{B}, \mathrm{c}-\mathrm{myc}, \mathrm{v}$-fes and ornithine decarboxylase (Fan et al, 1998; Duxbury and Whang, 2007). The mechanisms by which RRM2 regulates the function of these oncogenes remains unknown. As RRM2 contains a dinuclear iron center, which can produce a tyrosyl free radical transmitted to RRM1 for dNDP synthesis, its overexpression may result in increased radical generation and formation of reactive oxygen species (ROS). This hypothesis was partly supported by an in vitro study, which showed that recombinant human RRM2 protein had a pro-oxidant potential to oxidise carboxy-H2DCF (Xue et al, 2006). ROS has a critical role in tumour growth, metastasis and angiogenesis (Xia et al, 2007; Lee and Kang, 2013), and induces activation of mitogen-activated protein kinases, NF- $\kappa \mathrm{B}$ and activator protein 1 (Tian et al, 2012; Drigotas et al, 2013; Tsai et al, 2013), which are related to tumour progression. However, it is unclear whether ROS is involved in a malignancy potential mediated by RRM2. In the present study, we found that RRM2 was a novel downstream target activated by HPVE7, and promoted angiogenesis by producing ROS, activating the ERK1/2 signalling pathway and inducing expression of HIF- $1 \alpha$ and VEGF, leading to cervical angiogenesis and carcinogenesis. These findings identify a novel HPV E7-RRM2-ROS-ERK-VEGF pathway involved in HPVassociated cervical cancer development.

\section{MATERIALS AND METHODS}

Patient samples, cell culture and reagents. Human cervical cancer samples were collected from sixty patients diagnosed with invasive squamous cell cancer, undergoing resection of cervical cancer with an average age of $49 \pm 8.7$ years. This study was approved by local institutional review boards on human subject research and in accordance with the Declaration of Helsinki. Written informed consents were obtained from all study participants. Human cervical cancer cell lines (HeLa, CaSki and C33A) and breast cancer cell line (MCF-7) were obtained from Chinese Center for Type Culture Collection (Wuhan, China) and grown in Dulbecco's modified Eagle's medium supplemented with $10 \%$ fetal bovine serum (FBS, Gibco Life Technologies, Gaithersburg, MD, USA) in a humidified incubator with $5 \% \mathrm{CO} 2$ at $37^{\circ} \mathrm{C}$. The mouse monoclonal antibodies against RRM2 and HIF- $1 \alpha$ were purchased from Epitomics (Burlingame, CA, USA). Rabbit antibodies against phospho-ERK1/2 (Thr202/Tyr204), total-ERK1/2 and GAPDH, and U0126 (MEK1/2 Inhibitor) were obtained from Cell Signalling Technology (Beverly, MA, USA). Goat anti-HPV18E7, antiHPV18E6 and anti-CD31 antibodies were from Santa Cruz Biotech (Santa Cruz, CA, USA). A mouse anti-HPV16E6 antibody was from Abcam (Cambridge, MA, USA). Mouse anti- $\alpha$-tubulin and anti-p53 antibodies were from Millipore (Billerica, MA, USA). HRPconjugated secondary antibodies were from Sigma (St Louis, MO, USA). A complete protease inhibitor cocktail and $N$-acetylcysteine (NAC) were purchased from Roche (Indianapolis, IN, USA). Negative control (NC) siRNA were chemically synthesised from Guangzhou RioboBio (Guangzhou, Guangdong, China).

Gene silencing and overexpression. Inhibition of specific gene expressions was achieved by RNA interference using a mixture of four siRNAs (ON-TARGET plus SMARTpool; Dharmacon, Lafayette, CO, USA). Cells were transfected with $100 \mathrm{~nm}$ siRNAs using DharmaFECT (Dharmacon) according to the manufacturer's instructions. For overexpression studies, cells were transfected each plasmid DNA using Lipofectamine 2000 (Life Technologies Corporation, Gaithersburg, MD, USA).

Western blot analysis. The concentration of cell extracts was measured by a Bradford Protein Assay Kit (Beyotime). An equal amount of total protein extracts was separated by $12 \%$ SDS-PAGE and transferred to PVDF transfer membranes (Bio-Rad Laboratories, Hercules, CA, USA) and immunoblotted with various antibodies. The signals were detected by incubation with a SuperSignal West Pico Chemiluminescent detection system (Pierce Chemical Co, Rockford, IL, USA). Images were generated using a ChemiDoc XRS (Bio-Rad Laboratories), and protein band intensity was measured using Quantity One software. Three independent experiments were performed.

Quantitative real-time PCR analysis (qRT-PCR). Total RNA samples were extracted from cells using Trizol reagent (Invitrogen, Carlsbad, CA, USA). The RNA was converted into cDNAs using the RevertAid First Strand cDNA Synthesis Kit (Fermentas, K1621). Real-time PCR was performed using FastStart Universal SYBR Green Master kit (Roche Diagnostics) and analysed with an Applied Biosystems 7900 Real-Time PCR System. Primer sequences were as follows:VEGF, $5^{\prime}$-TCGAGTACATCTTCAAGC CATCCTG-3' and $5^{\prime}$-TCCTATGTGCTGGCCTTGGTGAG $3^{\prime}$; RRM2, $5^{\prime}$-TTTAGTGAGCTTAGCACAGCGGGA-3' and $5^{\prime}$-AA ATCTGCGTTGAAGCAGTGAGGC- $3^{\prime}$; $\beta$-actin, $5^{\prime}$-CATCGTCCA CCGCAAATG- $3^{\prime}$ and 5' ${ }^{\prime}$-CACCTTCACCGTTCCAGTT- $3^{\prime}$; GAPDH, $5^{\prime}$-TGAACGGGAAGCTCACTGG- $3^{\prime}$ and $5^{\prime}$-TCCACCACCCTGT TGCTGT $-3^{\prime}$. Fold changes in expression were calculated. The data was analysed using the method of $2^{-} \Delta \Delta$ relative expression quantity as described previously by us (Wang et al, 2011; Zhou et al, 2013).

Immunohistochemistry. Immunohistochemical staining of formaldehyde-fixed, deparaffinised tissue sections was carried out after antigen retrieval by treating the sections in a microwave oven for $10 \mathrm{~min}$ in $10 \mathrm{mmoll}^{-1}$ citric acid (pH6.0). CERVIMAXTM IHC kit 
(Adriacell S.p.A.) was used for immunohistochemical detection of HPVE7 protein within cervical cancer tissue sections as described (Ressler et al, 2007; Faoro et al, 2013). To detect RRM2, CD31 and phospho-ERK1/2 within Human cervical cancer tissue sections or mouse tumour sections, the primary antibody was incubated at $4{ }^{\circ} \mathrm{C}$ overnight, and horseradish peroxidase-conjugated secondary antibody was then added. The colour reaction was developed with 330-diaminobenzidine tetrahydrochloride $/ 0.03 \% \mathrm{H}_{2} \mathrm{O}_{2}$, followed by counterstaining with hematoxylin.

HPVE7, RRM2 and phospho-ERK1/2 staining was evaluated as a score of percentage of positive staining cells as follows: $(++)$, $\geqslant 50 \%$ of cells stained; $(+-),<50 \%$ of cells stained. Microvessel count was performed on three high-power fields $(\times 200)$ chosen within the whole-vascularised areas. Any endothelial cell or cluster of endothelial cells positive for CD31 was counted.

Luciferase assays. A DNA fragment containing a wild-type RRM2 $(-2147 /+1)$ promoter, a mutant RRM2 promoter (deletion of $-1581 /-1582$ and $-775 /-768$ from $-2147 /+1)$ or a p21 $(-2400 /+1)$ promoter (el-Deiry et al, 1995) was separately cloned into the luciferase report vector pGL4, which generated reporter genes $p R R M 2(W T)-L u c, p R R M 2(M U T)-L u c$ and $p 21-L u c$. Each reporter was co-transfected into MCF-7 cells with pGL4Renilla for normalisation, and other indicated expression constructs using Lipofectamine 2000. Forty-eight hours after transfection, firefly and renilla luciferase activities of cell lysates were analysed with Dual-Luciferase Reporter Assay kit (Gibco Life Technologies, Gaithersburg, MD, USA) according to the manufacturer's instructions. Each experiment was performed in triplicate and repeated at least three times.

Enzyme-linked immunosorbent assays. The concentration of VEGF in conditioned media was determined using a human VEGF ELISA kit according to the manufacture's instruction (R\&D Systems, Minneapolis, MN, USA). Optical densities were read by a microplate reader (Flexstation 3, Molecular Devices Corporation, Sunnyvale, CA, USA) at $405 \mathrm{~nm}$. Each experiment was performed in triplicate and repeated at least three times.

Capillary tube formation assays. Human umbilical vein endothelial cells (HUVECs) were obtained from Pricells (Wuhan, Hubei, China) and suspended in conditioned media and seeded on growth factor-reduced Matrigel (BD Biosciences, Franklin Lakes, NJ, USA) in a 96-well plate as described by Tian et al (2004). After $8 \mathrm{~h}$ of incubation, the number of mature, well-connected capillary tubes were manually counted under an inverted Nikon Eclipse Ti microscope (Nikon USA, Melville, NY, USA) and compared among different groups.

ROS detection. Intracellular ROS levels were measured using a ROS-specific fluorescent dye 5(6)-chloromethyl-2-7-dichlorodihydrofluorescence diacetate (DCFHDA, Molecular Probes, Eugene, OR, USA), followed by quantification of labelled cells by flow cytometry. Various transfected cells were treated with $10 \mu \mathrm{M}$ DCFHDA for $30 \mathrm{~min}$ at $37^{\circ} \mathrm{C}$. Stained cells were washed twice with PBS and the ROS levels were measured by using a flow cytometer (Beckman Coulter, Bohemia, NY, USA, FC500). Data from at least 50000 cells were collected for each sample.

In vivo tumour angiogenesis and growth assays. The animal protocol for in vivo tumour angiogenesis and growth assays was approved by the Ethics Committee of College of Life Science and Technology at Huazhong University of Science and Technology. C33A cells were transfected with pcDNA3.1-RRM2 or pcDNA3.1 empty vector using Lipofectamine 2000. Cells with stable overexpression of RRM2 (C33A-RRM2) and a comparable control cell line $(\mathrm{C} 33 \mathrm{~A}-\mathrm{V}$, transfected with an empty vector) were generated by G418 selection for 14 days. Four-week-old female BALB/c nude mice (Chinese Academy of Sciences, Shanghai, China) were inoculated subcutaneously with $5 \times 10^{6}$ C33A-RRM2 or C33A-V cells in the right flank. Five mice were used per group. Tumour growth was observed every 3 days for 32 days by measuring the two dimensional longest axis (a) and the shortest axis (b) with a caliper. At day 32, the animals were killed and tumours were excised, fixed in formalin and processed for further immunohistochemical analysis.

Statistical analysis. All data were presented as means \pm s.d. for experiments performed at least three times. Student's $t$ tests were used to analyse the differences of means between two different groups. Pearson's $\chi 2$-tests were used to compare categorical data. A $P$-value of $<0.05$ was considered to be statistically significant.

\section{RESULTS}

HPVE7 regulates the expression level of RRM2 in cervical cancer cells. To determine whether HPVE7 is the upstream activator of RRM2, we investigated RRM2 expression in cancer cells with knockdown of E6/E7 expression as an siRNA targeting to E7 may silence E6 as well (Tang et al, 2006) or E6 expression alone by western blotting and quantitative RT-PCR analyses. As shown in Figure $1 \mathrm{~A}$ and $\mathrm{B}$, the RRM2 protein level was significantly downregulated by knockdown of E6/E7 expression in either HeLa or CaSki cells, but not by knockdown of E6 alone. As a control, p53 protein showed strong upregulation as expected for knockdown of E6 expression. Similarly, knockdown of E6/E7, but not E6 alone, significantly inhibited expression of the RRM2 mRNA (Figure 1A and $\mathrm{B}$ ). These results suggest that HPVE7 regulates expression of RRM2. To further establish the finding that HPVE7 regulates RRM2 expression, we transiently transfected HPV-negative C33A cervical cancer cells with an expression plasmid for E7 (pcDNA3.1E7) or E6 (pcDNA3.1E6). Analysis of RRM2 protein and mRNA expression showed that overexpression of E7, but not of E6, significantly enhanced RRM2 expression (Figure 1C).

To provide in vivo evidence that HPVE7 regulates expression of RRM2, we examined the expression levels of HPVE7 using the CERVIMAXTM IHC kit, which contains a mixture of highly purified monoclonal antibodies against those high-risk E7 proteins and RRM2 in sixty human primary cervical cancer specimens by immunohistochemical (IHC) staining. We found that the expression level of RRM2 was high in 31 of 38 specimens with high HPVE7 expression, but only in 5 of 22 specimens with low HPVE7 expression $(P<0.01)$ (Figure $1 D)$. The IHC data suggest a significant positive correlation between HPVE7 and RRM2 expression levels in cervical cancer. Together, these data indicate that RRM2 represents a novel target gene that is upregulated by HPVE7 in cervical cancer.

Regulation of RRM2 by HPVE7 is on the transcriptional level. As two highly conserved E2F-binding sites were identified within RRM2 promoter (Figure 2A), we investigated whether HPVE7 directly activates transcription of RRM2. We co-transfected a RRM2 promoter-controlled luciferase reporter gene and an expression plasmid for either HPV16E7 or HPV18E7 in MCF-7 cells. Luciferase assays showed that overexpression of both HPV16E7 and HPV18E7 proteins resulted in transcriptional activation of the RRM2 promoter (Figure 2B). In contrast, overexpression of either HPV16E6 or HPV18E6 did not affect RRM2 promoter activity. As expected, both HPV16E6 and HPV18E6 significantly inhibited the activity of the p21 promoter, which is a transcriptional target for p53 (Figure 2C). These data indicate that high-risk HPVE7 specifically had a critical role in transcriptional activation of the RRM2 promoter.

As the ability of HPVE7 to bind to and inactivate $\mathrm{pRb}$ contributes to E7-mediated oncogenesis in the cervix (Boyer et al, 1996), we investigated whether E7-mediated activation of the RRM2 promoter was mediated by repression of $\mathrm{pRb}$. We assessed whether 
A

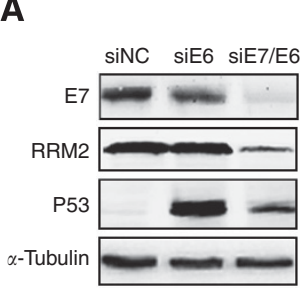

B

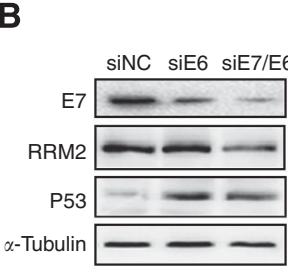

C

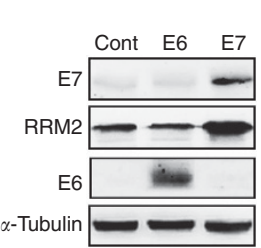

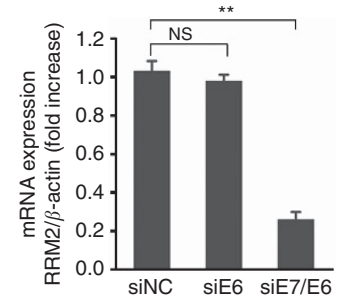
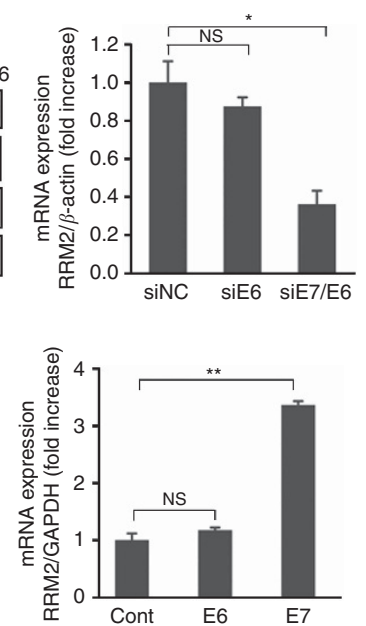

D

\begin{tabular}{|c|c|c|c|c|}
\hline \multicolumn{2}{|c|}{\begin{tabular}{c} 
Cervical cancer \\
\multirow{2}{*}{ Tissues }
\end{tabular}} & \multicolumn{2}{|c|}{ High risk HPV18E7 } & \multirow{2}{*}{ Total } \\
\cline { 2 - 4 } & Low $(+/-)$ & High $(+/+)$ & \\
\hline \multirow{2}{*}{ RRM2 } & Low (+/-) & 17 & 7 & 24 \\
\cline { 2 - 4 } & High (+/+) & 5 & 31 & 36 \\
\hline \multicolumn{2}{|c|}{ Total } & 22 & 38 & 60 \\
\hline
\end{tabular}

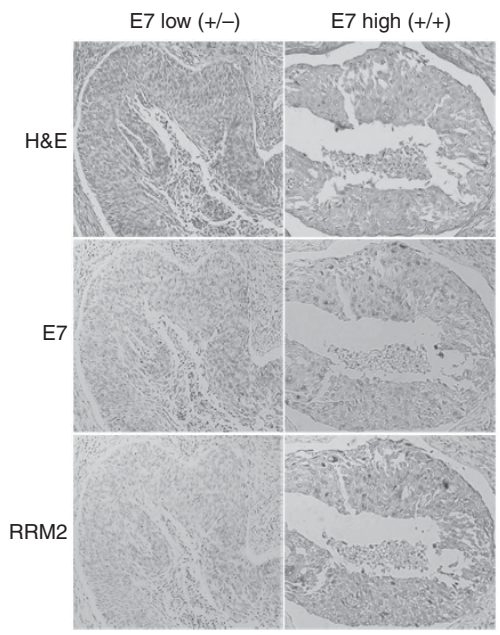

Figure 1. HPVE7 upregulates expression of RRM2. (A) Western blot (left panel) and real-time RT-PCR analyses of RRM2 in HeLa cells with knockdown of E6 or E6/E7 expression. siNC, negative control siRNA; siE6, siRNA against HPV18 E6; siE7/E6, siRNA against E6 and E7; p53, positive control for siRNA against E6; $\alpha$-tubulin, loading control for western blot analysis. GAPDH was used as an internal control for quantitative RT-PCR analysis. (B) Western blot (left panel) and quantitative real time RT-PCR analyses of RRM2 in CaSki cells with knockdown of E6 or E6/E7 expression. (C) Western blot (left panel) and quantitative real-time RT-PCR analyses of RRM2 in C33A cells with overexpression of E6 or E7. Cont, transfection with a pcDNA3.1 empty vector as control. (D) The expression level of RRM2 positively correlates with the level of the HPVE7 protein in human cervical cancer (upper panel). The expression levels of RRM2 and HPVE7 in 60 human primary cervical cancer specimens were measured by immunostaining with antibodies specific to RRM2 and HPVE7, and correlated. Positive correlation between RRM2 and HPVE7 levels were determined using a Pearson Chi-Square analysis $(P<0.01)$. The lower panel shows immunostaining images of two representative specimens, one with low $(+/-)$ and the other with high $(+/+)$ expression of RRM2 and HPVE7. H\&E staining served as control. The data are shown as mean \pm s.d. of three separate experiments. NS, no significant difference; ${ }^{*} P<0.05 ;{ }^{\star \star} P<0.01$.

a pRb-binding-deficient HPVE7 protein (Huh et al, 2005) was unable to transactivate the RRM2 promoter. The mutant HPVE7 protein failed to transactivate the RRM2 promoter (Figure 2D), suggesting that interaction between HPVE7 and pRB is essential for transcriptional activation of RRM2. To further determine the requirement of E2F sites for HPVE7-induced RRM2 promoter activity, we explored the effect of HPVE7 on a mutant RRM2 promoter luciferase reporter with the putative E2F-binding sites deleted. The mutant RRM2 promoter failed to elicit a response to HPVE7 (Figure 2E and F). Together, these data indicate that HPVE7 upregulates RRM2 transcription through interaction with $\mathrm{pRb}$ and direct binding of E2F to the RRM2 promoter region.

HPVE7-induced RRM2 expression upregulates VEGF expression in cervical cancer. Having established that RRM2 is a novel downstream target of HPVE7, we next investigated whether RRM2 is involved in HPVE7-mediated cervical carcinogenesis. Cervical cancer angiogenesis has been correlated with tumour progression and metastasis (Landt et al, 2011; Liu et al, 2011). RRM2 has recently been suggested to promote tumour angiogenesis by upregulating VEGF expression in oropharyngeal carcinoma cells. We hypothesised that RRM2 also regulated VEGF expression in cervical cancer cells. We measured the level of secreted VEGF by ELISA in C33A and HeLa cells. Transient overexpression of RRM2 by transfection led to a significant increase in VEGF secretion in the conditioned media from both cell lines compared with mocktransfection control or control transfection with an empty vector
(Figure 3A). The results were further supported by quantitative real-time RT-PCR analysis, which showed that overexpression of RRM2 significantly increased the expression level of the VEGF mRNA as compared with controls (Figure 3A). To determine whether RRM2-mediated upregulation of VEGF expression is further regulated by HPVE7, C33A cells were transfected with a HPV16E7 expression construct, followed by co-transfection with a RRM2 siRNA or a control siRNA. HPVE7-induced elevation of RRM2 protein expression was partially inhibited by co-transfection with the RRM2 siRNA but not by the control siRNA (data not shown). The increased VEGF protein secretion and mRNA expression by HPV16 E7 (siNC + E7) were significantly inhibited by co-transfection with the RRM2 siRNA (siRRM2 + E7) to a level without HPVE7 overexpression (siRRM2 + Vector) (Figure 3B). Knockdown of HPVE7 expression by an E7-specific siRNA led to significantly decreased VEGF protein secretion and VEGF mRNA expression compared with a control siRNA (siE7 + Vector $v s$ siNC, Figure 3C). In contrast, decreased VEGF protein secretion and mRNA expression caused by HPVE7 knockdown were significantly reversed by co-transfection with a RRM2 expression construct compared with empty vector control in HeLa cells (Figure 3C). These results suggest that RRM2 mediates HPVE7induced upregulation of VEGF expression.

RRM2 mediates capillary tube formation induced by expression of HPVE7 in cervical cancer cells. Because HPVE7 induces upregulation of VEGF expression by increasing RRM2 expression, 


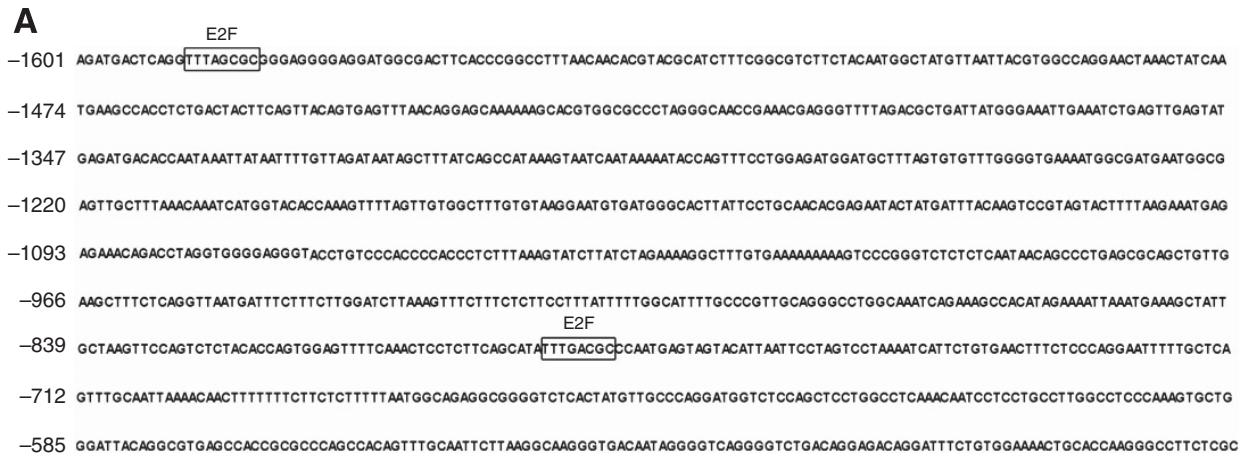

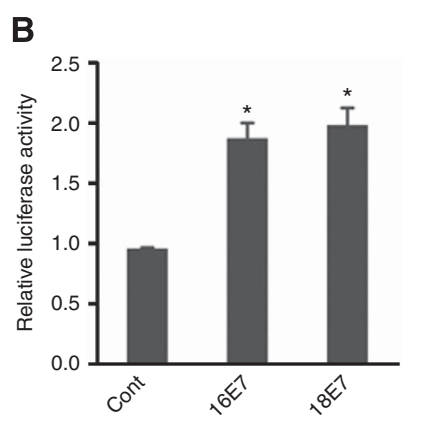
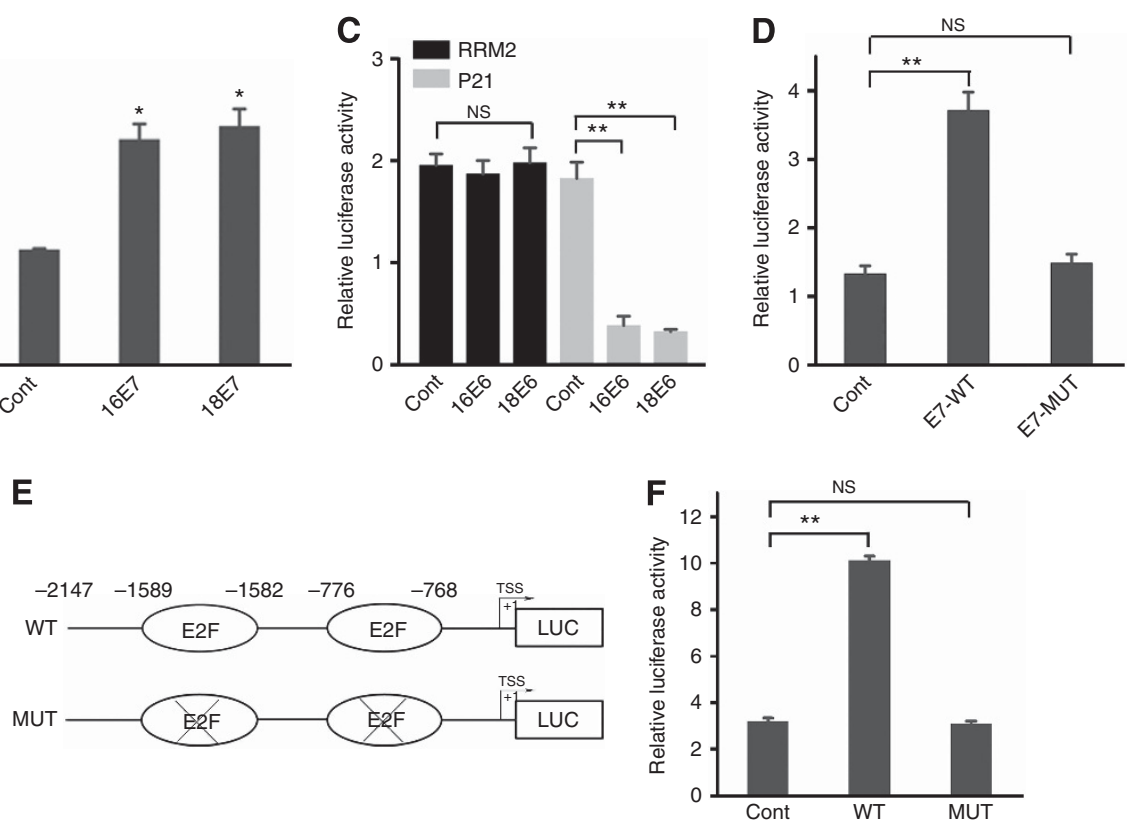

Figure 2. HPVE7 induces increased transactivation of the RRM2 promoter. (A) Sequence of the human RRM2 promoter region. (B) MCF-7 cells were co-transfected with a RRM2 promoter luciferase reporter, an expression plasmid for either HPV-16 E7 or an HPV-18 E7 (an empty vector as control, Cont), and a Renilla luciferase plasmid as an internal control. Twenty-four hours after transfection, cells were collected and used for measuring luciferase activities. The data are shown as mean \pm s.d. from triplicate wells. The experiment was repeated three times. (C) Luciferase assays for the RRM2 promoter (black columns) and the p21 promoter (white columns) in MCF-7 cells with overexpression of HPV16 E6, HPV18 E6 or with empty vector. (D) Luciferase assays for the RRM2 promoter in MCF-7 cells with overexpression of wild-type HPV16 E7 or mutant HPV16 E7 (E7-MUT), which lose the ability to bind to pRb. Cont, empty vector control. (E) Schematic diagram of the RRM2 promoter luciferase reporter genes. WT, wild type RRM2-Luc reporter; MUT, mutant RRM2-Luc with two consensus E2F-binding sites deleted. (F) Luciferase assays for either the wild-type RRM2 promoter (WT) or a mutant RRM2 promoter with a deletion of the E2F-binding site (MUT) in MCF-7 cells with overexpression of HPV-16 E7. Cont, empty vector control. NS, no significant difference; ${ }^{\star} P<0.05,{ }^{\star \star} P<0.01$.

we characterised the functional role of HPVE7 and RRM2 in cervical cancer angiogenesis using an in vitro matrigel-based capillary tube formation assay by HUVECs. As shown in Figure 4A, HUVECs formed significantly more mature, wellconnected tubes in conditioned media from C33A cells with overexpression of HPV16E7 compared with mock-transfection control. Increased HUVECs tube formation by overexpression of HPVE7 was significantly abrogated by co-transfection with a RRM2-specific siRNA compared with a control siRNA (Figure 4A). These data suggest that RRM2 mediates HPVE7induced HUVEC capillary tube formation. To confirm the role of VEGF in RRM2-regulated cervical cancer cell angiogenesis, we performed a VEGF-blocking assay. HUVECs formed significantly more capillary tubes in conditioned media from C33A cells with overexpression of RRM2 (compare RRM2 to Cont), but the effect was abrogated by incubation with a sufficient amount of VEGF antibody (compare anti-VEGF + RRM2 to IgG + RRM2) (Figure 4B). These data suggest that RRM2 induced cervical cancer angiogenesis is mediated at least partly by VEGF.
RRM2 expression correlates with microvessel densities in cervical cancer. Because the results above indicate that RRM2 positively regulates cervical cancer angiogenesis in vitro, we next investigated human primary tumours to determine whether there was any correlation between RRM2 expression and microvessel formation in cervical cancer specimens in vivo. The sections from 60 cervical cancer samples were subjected to immunohistochemical analysis for RRM2 expression and microvessel densities. On the basis of the expression level of RRM2, we divided the 60 cervical cancer samples into two groups. Among 60 samples, $60 \%$ showed strong expression of RRM2, whereas $40 \%$ displayed weak expression of RRM2. The tumour sections were stained with an anti-CD31 antibody, and the number of CD31-positive signals per area in each section was counted. As shown in Figure $4 \mathrm{C}$, the density of CD31 vessels was significantly higher in the group with strong RRM2 expression than that with weak RRM2 expression. These results are consistent with the data from in vitro capillary tube formation assays. Together, we conclude that RRM2 has a vital role in HPVE7-induced cervical cancer angiogenesis. 

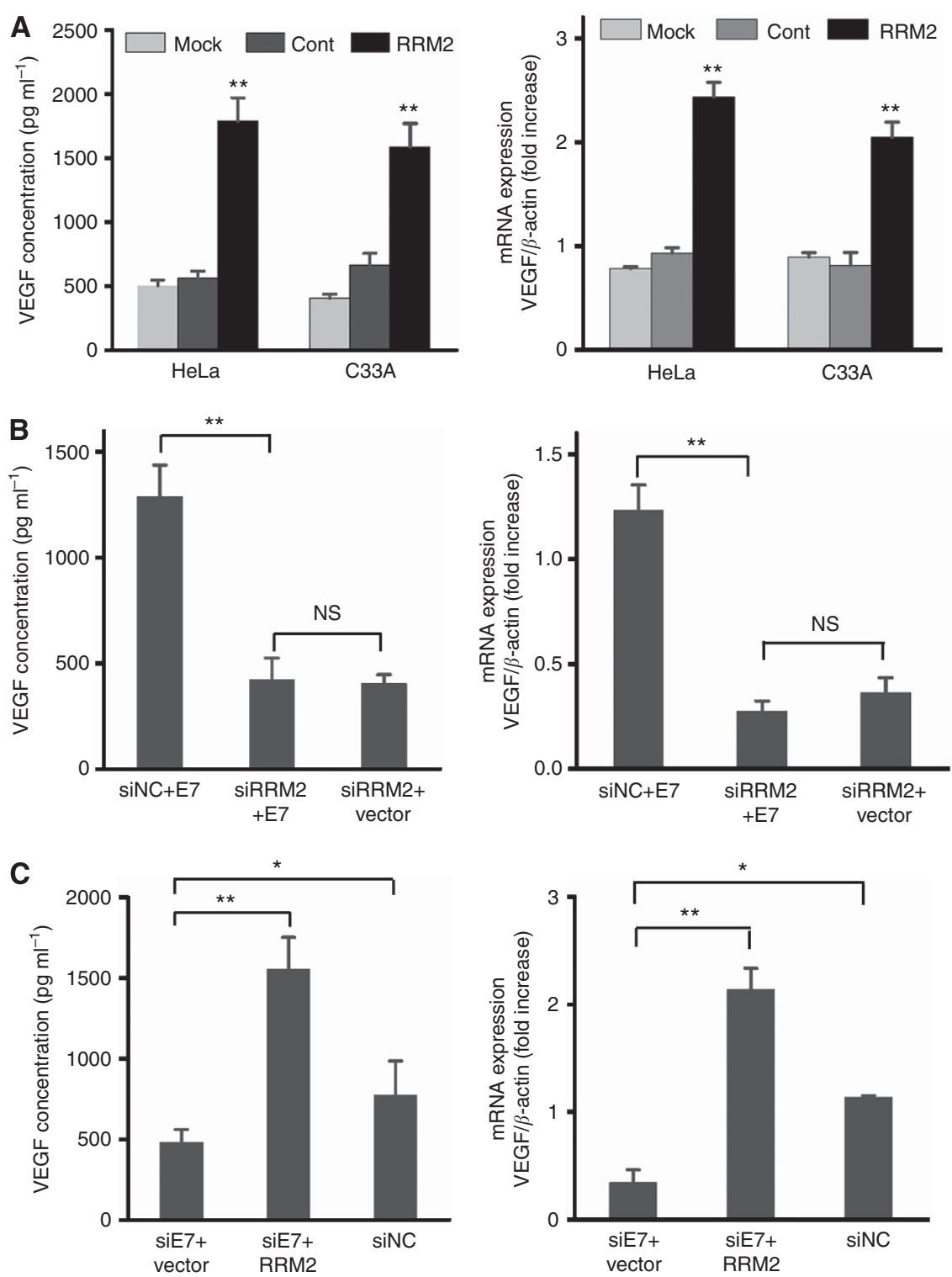

Figure 3. RRM2 mediates HPVE7-induced VEGF expression in cervical cancer cells. (A) C33A and HeLa cells were transiently transfected with a RRM2 expression plasmid. Cells with mock transfection or transfected with the pcDNA3.1 empty vector served as controls. Left panel, the level of secreted VEGF in the conditioned media derived from transfected C33A or HeLa cells was determined by ELISA. Right panel, the expression level of VEGF mRNA in transfected cells was determined by quantitative real time RT-PCR. The data are shown as mean \pm s.d. from triplicate wells. The experiment was repeated three times. (B) C33A cells were transfected with a HPV16 E7 expression plasmid, and a RRM2 siRNA or a control siRNA. Left panel, ELISA data for the level of secreted VEGF in the conditional media from transfected cells. Right panel, the expression level of VEGF mRNA was determined by quantitative real time RT-PCR. (C) HeLa cells were transfected with a HPV18 E7 siRNA or a control siRNA followed by cotransfection with a RRM2 expression plasmid or the control empty vector. Left panel, ELISA data for the level of secreted VEGF in the conditional media from transfected cells; right panel, quantitative real time RT-PCR data for the expression level of VEGF mRNA. NS, no significant difference; ${ }^{\star} P<0.05,{ }^{* \star} P<0.01$.

RRM2 mediates HPVE7-induced activation of the ERK1/2 pathway and increased expression of HIF-1 $\alpha$ and VEGF. The molecular mechanism by which RRM2 induces upregulation of VEGF is unknown. A previous study has indicated that HPVE7 can promote HIF- $1 \alpha$ protein accumulation and VEGF expression via activation of the ERK1/2 signalling pathway (Tang et al, 2007). Our data above suggested that RRM2 mediated HPVE7-induced upregulation of VEGF expression. We speculated that RRM2 as a downstream target for HPVE7 might also elevate HIF-1 $\alpha$ and VEGF expression through the ERK1/2 pathway. To validate this hypothesis, we transfected C33A cells with a RRM2 expression vector, followed by treatment with U0126 $(10 \mu \mathrm{M})$, an inhibitor for MEK1 (the upstream activator of ERK1/2). Overexpression of
RRM2 induced an elevated level of phosphorylated ERK1/2 accompanied with upregulation of HIF- $1 \alpha$ expression and VEGF secretion, but treatment with U0126 led to a significant decrease in RRM2-induced increases of HIF- $1 \alpha$ expression and VEGF secretion (Figure $5 \mathrm{~A}$ and $\mathrm{B}$ ). To further investigate whether RRM2 is involved in HPVE7-induced activation of ERK1/2 and increases of HIF- $1 \alpha$ expression and VEGF secretion, we performed RRM2 blocking and RRM2 rescue assays. We found that upregulation of phosphorylated ERK1/2, HIF- $1 \alpha$ and VEGF expression induced by HPVE7 in C33A cells was significantly abrogated by co-transfection with a RRM2 siRNA, but not by a control siRNA (Figure 5C). In contrast, downregulation of phosphorylated ERK1/2, HIF- $1 \alpha$ and VEGF expression induced 
A

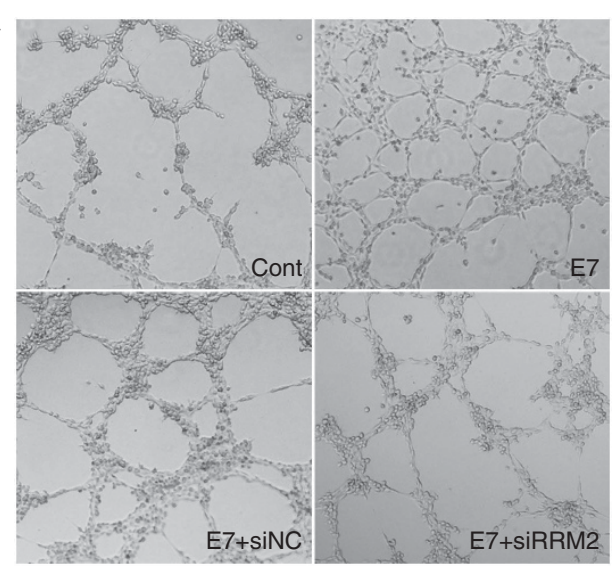

B

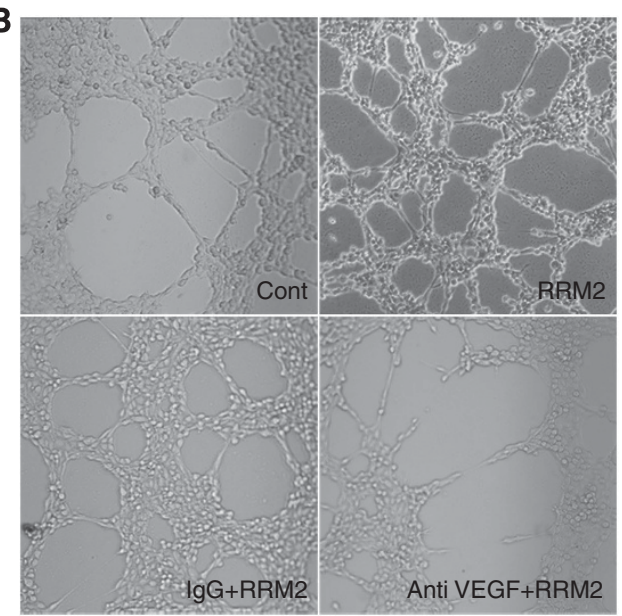

C

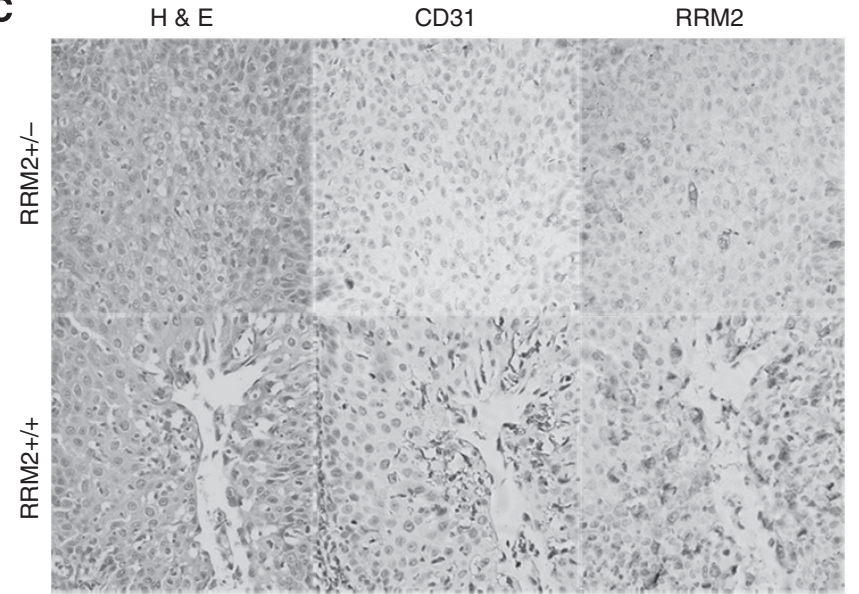

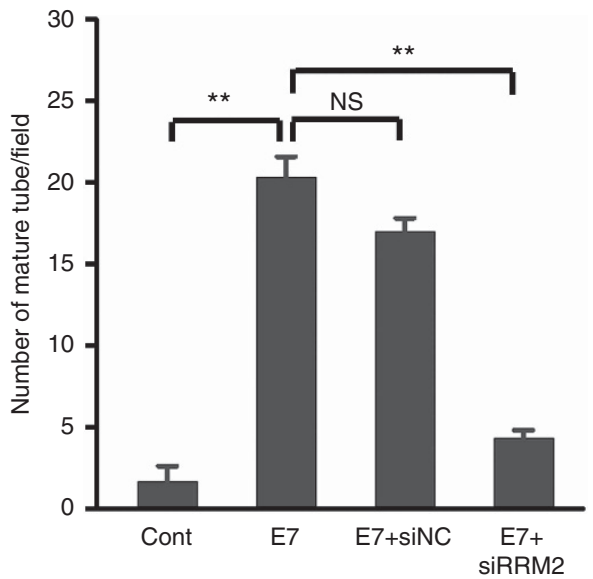

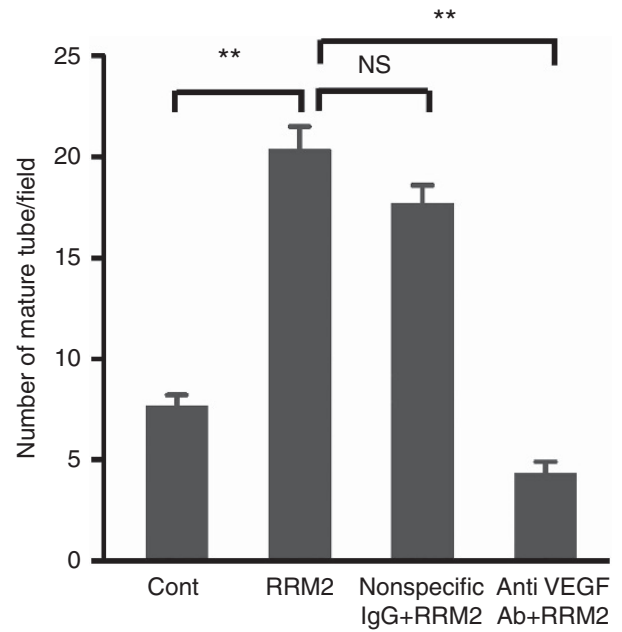

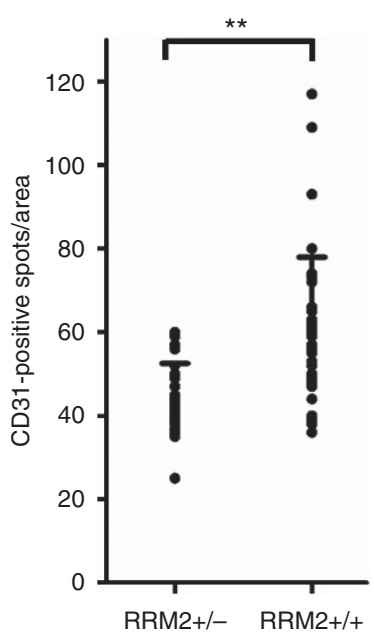

Figure 4. RRM2 is involved in cervical cancer angiogenesis in vitro and in vivo. (A) Results from in vitro matrigel-based angiogenesis assays by HUVECs with conditioned media from C33A cells transfected with pcDNA-HPV18 E7 with or without RRM2 siRNA. Representative images (left panel) and mean numbers of mature tube-forming structures (right panel) at $\times 100$ magnification. HUVECs $\left(1 \times 10^{4}\right.$ cells per well) were seeded onto the surface of 96 -well cell culture plates precoated with matrigel and then incubated at $37^{\circ} \mathrm{C}$ for $8 \mathrm{~h}$ in the conditioned media derived from transfected C33A cells. The data presented are representative of three separate experiments. (B) Application of a VEGF antibody (400 $\mathrm{ng} \mathrm{ml}^{-1}$ ) to neutralise VEGF in three conditioned media abrogated RRM2-enhanced capillary tube formation. (C) Immunohistochemical analysis of 60 cervical cancer samples stained with anti-RRM2 and anti-CD31 antibodies (left panel). Representative images of weak RRM2 expression samples ( + / - ; $n=24)$ and strong RRM2 expression samples $(+/+; n=36)$ are shown. H\&E staining served as control. Original magnification, $\times 100$. The data on CD31-positive microvessel densities were plotted in the right panel. Microvessel densities were significantly higher in cancer samples with strong RRM2 expression than that with weak expression. Bars indicate the mean number of vessels. ${ }^{\star \star} P<0.01$.

by HPVE7 knockdown in HeLa cells was significantly reversed by co-transfection with a RRM2 expression plasmid, but not by a control empty vector (Figure 5D). Taken together, these results suggest that RRM2, as an important target gene for HPVE7, is involved in HPVE7-induced activation of ERK1/2 and increases of HIF- $1 \alpha$ and VEGF expression. 

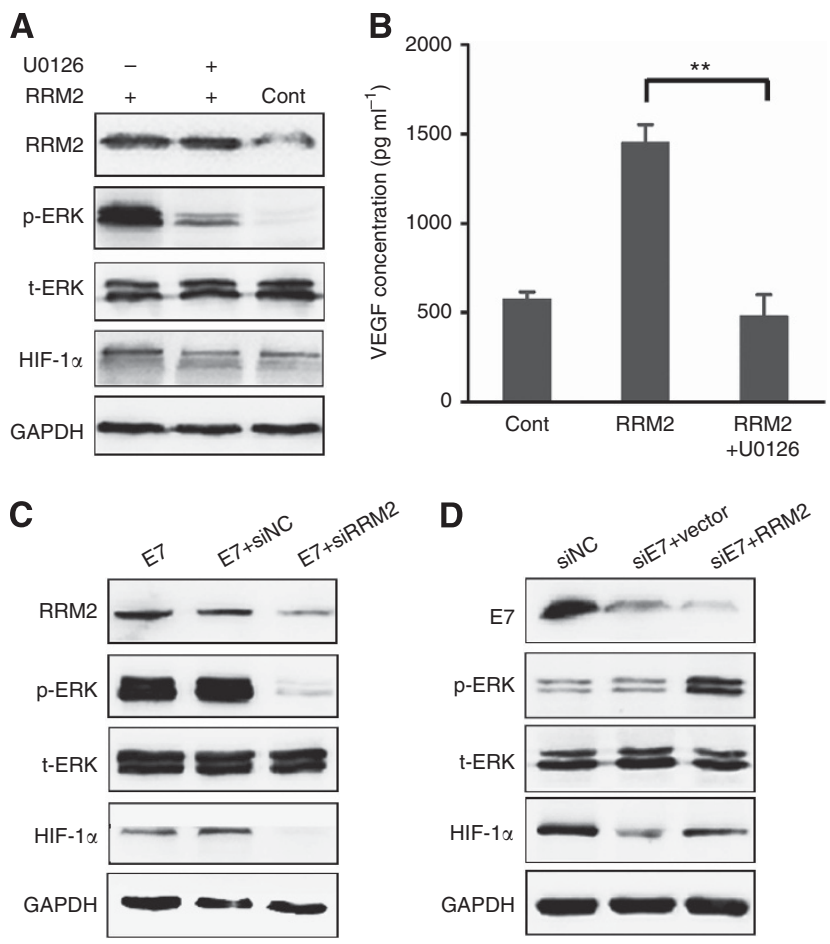

Figure 5. RRM2 mediates HPVE7-induced activation of the ERK1/2 signalling pathway and increases of HIF- $1 \alpha$ and VEGF expression. (A) RRM2-induced HIF-1 $\alpha$ protein expression was abolished by inhibition of the ERK1/2 signalling pathway by U0126. C33A cells transfected with a RRM2 expression plasmid or a control empty vector were treated with $10 \mu \mathrm{M}$ U0126 or vehicle control for $16 \mathrm{~h}$. The expression levels of phosphorylated ERK $1 / 2$ and HIF- $1 \alpha$ were examined by western blot analysis. t-ERK, total ERK levels used as loading control; GAPDH, another loading control. (B) RRM2-induced VEGF expression was abolished by inhibition of the ERK1/2 signalling pathway by $U 0126$. The level of secreted VEGF in the conditioned media was measured by an ELISA assay. The data presented are representative of three independent experiments. (C) C33A cells were transfected with PCDNA-HPV16E7, followed by co-transfection with a RRM2 siRNA (siRRM2) or a control siRNA (siNC) for another $24 \mathrm{~h}$. Cell lysates were used for western blot analysis for phosphorylated ERK $1 / 2$ and HIF-1 $\alpha$ protein levels. (D) HeLa cells were transfected with a HPV18 E7 siRNA (siE7) or a control siRNA (siNC), followed by co-transfection with pcDNA-RRM2 for another $24 \mathrm{~h}$. Cell lysates were used for western blot analysis for phosphorylated ERK1/2 and HIF-1 $\alpha$ protein levels. $\star \star P<0.01$.

RRM2 induced activation of ERK1/2 and increases of HIF- $1 \alpha$ and VEGF expression via ROS production. Next, we investigated the underlying mechanism by which RRM2 modulates the activation of ERK1/2 and increases HIF- $1 \alpha$ and VEGF expression. Previous studies have shown that RRM2 can induce ROS production in vitro (Xue et al, 2006), and ROS can activate several signalling pathways including the ERK1/2 pathway and regulate HIF- $1 \alpha$ and VEGF expression (Meng et al, 2012). We therefore speculated that ROS could be involved in the activation of ERK1/2 and enhancement of HIF- $1 \alpha$ and VEGF expression in cervical cancer cells by RRM2. To test whether RRM2 could affect the intracellular ROS level in cervical cancer cells, we used the fluorogenic dye DCFHDA. Transfection of a RRM2 expression plasmid significantly increased the endogenous ROS level in C33A cells as determined by flow cytometry analysis. In contrast, the endogenous ROS level was significantly decreased in HeLa cells transfected with a RRM2 siRNA (Figure 6A). These results suggest that RRM2 can induce ROS production in cervical cancer cells.
A
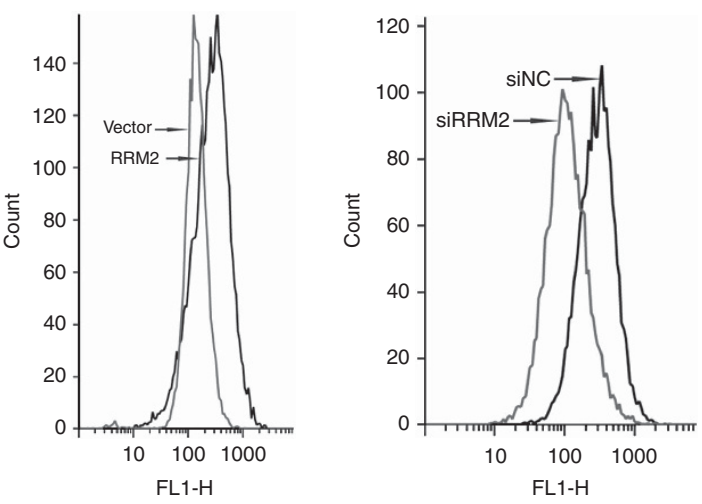

B
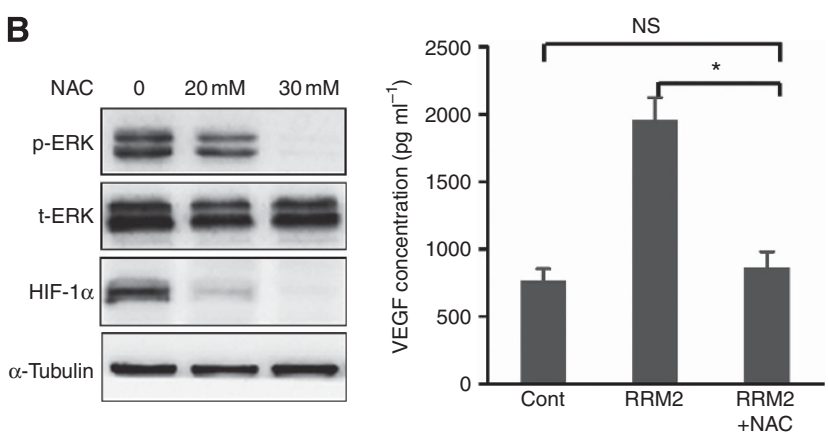

Figure 6. RRM2 elicited production of ROS to activate the ERK1/2 pathway, and enhance HIF-1 $\alpha$ and VEGF expression. (A) RRM2 increases generation of ROS in cervical cancer cells. C33A or HeLa cells were transfected with a RRM2 expression plasmid (empty vector as control, left panel) or a RRM2 siRNA (siNC, negative control siRNA, right panel) for $48 \mathrm{~h}$. Cells were then incubated with $20 \mu \mathrm{m}$ DCFHDA in the dark for 30 min at $37^{\circ} \mathrm{C}$, washed in PBS, resuspended in PBS and analysed by flow cytometry. (B) NAC prevents RRM2 induced activation of ERK1/2 ( $p$-ERK) and increases of HIF-1 $\alpha$ and VEGF expression. C33A cells were transfected with a RRM2 expression plasmid for $24 \mathrm{~h}$,

followed by treatment with an indicated amount of NAC for $24 \mathrm{~h}$. Cells were lysed and used for western blot analysis with antibodies against pERK1/2 and HIF-1 $\alpha$. t-ERK, total ERK levels used as loading control; $\alpha$-tubulin, loading control. The expression level of VEGF in conditioned media was measured by an ELISA assay. ${ }^{\star} P<0.05$.

We next investigated the effect of NAC (N-acetylcysteine, $10 \mathrm{mmoll}^{-1}$ ), a ROS scavenger, on the activation of ERK1/2 and upregulation of HIF- $1 \alpha$ and VEGF that were induced by RRM2 overexpression. We found that RRM2-mediated ERK1/2 phosphorylation and enhancement of HIF- $1 \alpha$ and VEGF expression were significantly attenuated by NAC, but not by the vehicle control (Figure 6B). Therefore, activation of ERK1/2 and upregulation of HIF- $1 \alpha$ and VEGF by RRM2 is dependent on the generation of intracellular ROS.

RRM2 enhances growth and angiogenesis of xenograft tumours in vivo. To further investigate the role of RRM2 in tumorigenesis in vivo, C33A cells with and without stable expression of RRM2 were inoculated subcutaneously into the flank of nude mice and tumour growth was monitored and compared. Tumours from C33A cells with overexpression of RRM2 displayed a significantly larger mass than that from cells without overexpression of RRM2 (Figure 7A and B). Quantitative RT-PCR analysis showed that expression of RRM2 and VEGF was significantly higher in the RRM2 overexpression xenografts than in xenografts without RRM2 overexpression (Figure 7C). These data showed that overexpression of RRM2 promoted tumour growth through VEGF in vivo. Then, we explored whether RRM2 overexpression affected 
A

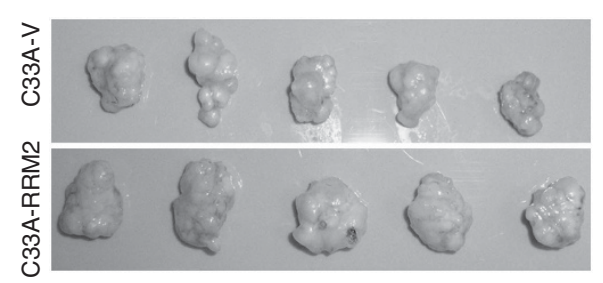

B

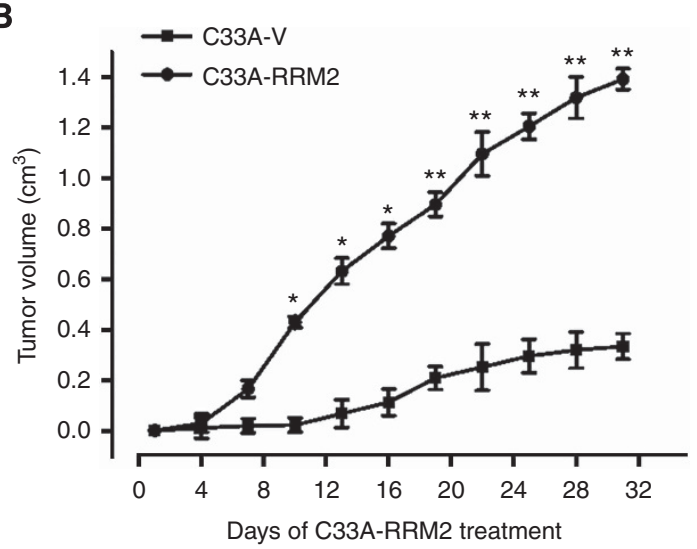

D

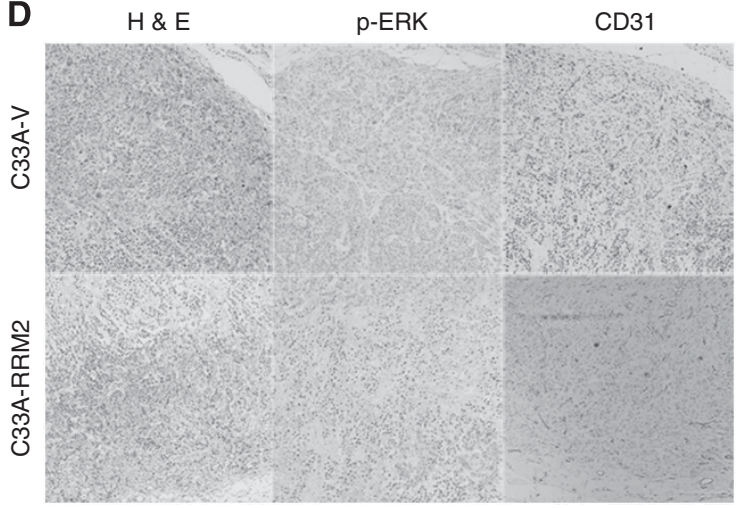

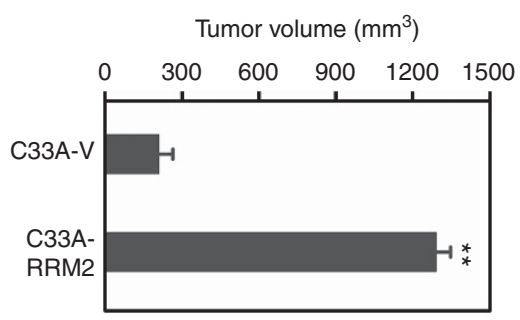
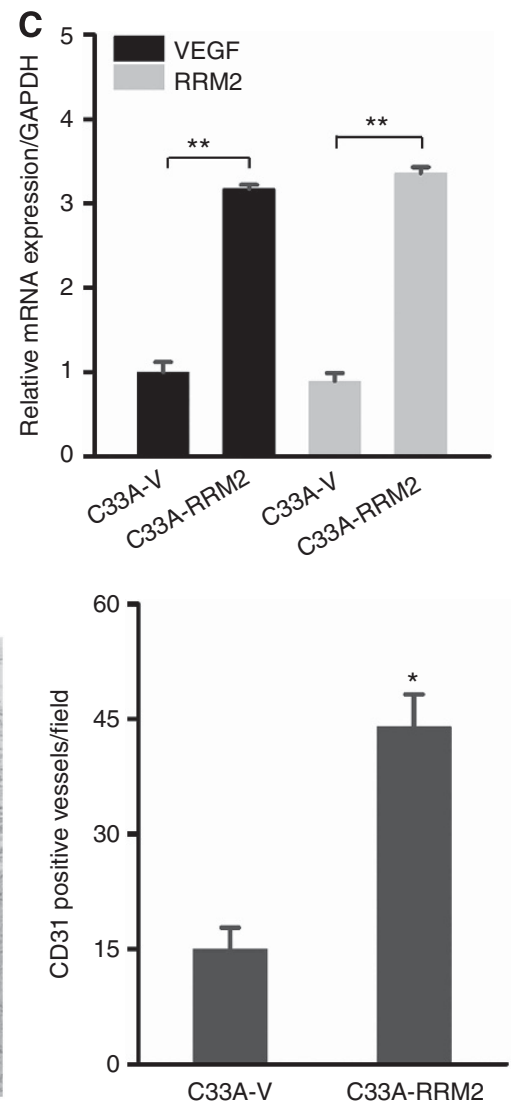

Figure 7. RRM2 overexpression promotes the growth and angiogenesis of cervical cancer xenografts in nude mice. (A) C33A-RRM2 and C33Avector cells were subcutaneously inoculated into the flank of nude mice. Thirty-two days after the inoculation, the mice were killed and tumour tissues were collected. Left panel shows photographs of the tumours. Right panel shows that overexpression of RRM2 increased the tumour size of C33A-RRM2 at the end of experiment. (B) Overexpression of RRM2 significantly enhanced tumour growth as compared with empty vector.

The graph shows tumour growth curves in nude mice inoculated with C33A cells with overexpression of RRM2 or cells with the cloning vector. Data are presented as means \pm s.d. $(n=5)$. (C) quantitative RT-PCR analysis of RRM2 and VEGF mRNA in C33A-RRM2 and C33A-vector xenografts. Data are shown as mean \pm s.d. from five mice. (D) Representative images of tumour sections from RRM2-overexpressed cells and control cells immunostained with pERK1/2 and CD31 antibodies (left panel). The level of phosphorylated ERK1/2 was significantly higher in RRM2overexpression tumours than in control tumours. H\&E staining served as control. Right panel, CD31-positive vessel densities were significantly increased in C33A-RRM2 tumour xenografts compared with that from C33A-vector. Data are presented as mean \pm s.d. $(n=5)$. ${ }^{\star} P<0.05,{ }^{\star \star} P<0.01$.

the in vivo level of phosphorylated ERK1/2 and angiogenic potential of tumour cells. We performed immunohistochemistry on the xenograft tumours for pERK1/2 and CD31, and the number of CD31-positive vessels per tumour was counted. The tumour xenografts derived from cells with RRM2 overexpression had a significantly higher level of pERK and number of CD31 positive vessels than xenografts without RRM2 overexpression. Mean vessel counting per field was $42 \pm 6.2$ for xenografts with RRM2 overexpression $(n=5)$ and $22 \pm 4.8$ for xenografts without RRM2 overexpression $(n=5)$, and the difference was statistically significant (Figure 7D). These data suggest that the enhanced tumour growth observed in xenografts with RRM2 overexpression was at least partly due to increased angiogenesis, which increases the capacity of blood vessels to supply the tumours with nutrients and oxygen.

\section{DISCUSSION}

HPVs E6 and E7 are critically involved in the development of cervical cancer, however, the molecular regulatory mechanisms downstream of these two oncoproteins are not fully elucidated. The present study identified RRM2 as a novel downstream target regulated by HPVE7. The conclusion is supported by the following evidences: RRM2 expression was reduced by knockdown of HPVE7 and increased by overexpression of HPVE7 (Figure 1); HPVE7 can upregulate RRM2 expression and activate the RRM2 promoter (Figure 2).

The present study elucidated the detailed molecular mechanism by which HPVE7 upregulates the expression of RRM2. 
Luciferase assays showed that HPVE7 upregulated RRM2 expression by activating RRM2 promoter (Figure 2). Activation of the RRM2 promoter by HPVE7 requires the interaction between HPVE7 and pRB because a mutant HPVE7 with a deletion of the $\mathrm{pRb}$ binding motif failed to transactivate the RRM2 promoter (Figure 2). Activation of the RRM2 promoter by HPVE7 also requires the binding of E2F to the promoter region because a mutant RRM2 promoter lacking the E2F response element could not be activated by HPVE7. Moreover, in line with the results from ex vivo cellular studies, we found that the RRM2 expression patterns in vivo significantly correlated with that of HPVE7 in a series of cervical cancer samples. On the basis of these results, we propose a working model for activation of RRM2 by HPVE7. Interaction between E7 and $\mathrm{pRb}$ leads to the release of E2F from the $\mathrm{pRb}$ complex. The released E2F then binds to the RRM2 promoter region and activates the transcription of RRM2. The model is strongly supported by previous reports that HPVE7 can inactivate $\mathrm{pRb}$ protein function through its ubiquitination-mediated degradation, which leads to the release of E2F and activation of E2F-regulated genes (Katich et al, 2001; Holland et al, 2008).

In this study, we further investigated the functional consequences of RRM2 overexpression in cervical cancer. Interestingly, we found that RRM2 overexpression produces ROS, which leads to the activation of the ERK1/2 signalling pathway, increased expression of HIF- $1 \alpha$ and VEGF, increased angiogenesis and eventually cervical carcinogenesis. Enhanced angiogenesis is tightly associated with the progression and metastasis of cervical cancer (Landt et al, 2011; Liu et al, 2011). There was significant correlation between VEGF expression levels and high-risk HPV infection in the progression of cervical cancer (Branca et al, 2006). Previous studies have shown that RRM2 can promote tumour angiogenesis by upregulating VEGF expression in some cancer cells. Thus, we hypothesised that RRM2 might be functionally involved in the progression of cervical cancer partly via enhanced angiogenesis by increasing VEGF expression. The hypothesis was verified as we showed for the first time that RRM2 upregulated the expression of HIF- $1 \alpha$, which is an important modulator for VEGF transcription, and resulted in a significant increase in VEGF expression in cervical cancer cells. Our data from clinical samples also showed that higher RRM2 expression was correlated with higher microvessel densities in cervical cancer patients. Furthermore, xenograft studies in immunocompromised nude mice indicated that the tumours from the RRM2 overexpression group had increased VEGF expression and blood vessel formation compared with the control group. Moreover, we found that RRM2 promoted cervical cancer angiogenesis was VEGF dependent, as RRM2 stimulated in vitro capillary tube formation was suppressed by a VEGF neutralising antibody. Together, our findings from both in vitro and in vivo studies suggested that RRM2 might be implicated in cervical cancer development at least partly through enhancement of angiogenesis. Consistent with a previous study showing that $\mathrm{pRb}$ can regulate the expression of angiogenic factors, such as VEGF, through an E2F-depedent mechanism (Gabellini et al, 2006), our findings provided strong evidence that RRM2, as an E2F-regulated gene, might be involved in E2F-mediated angiogenesis.

Zhang et al (2009) reported that RRM2 could modulate VEGF expression, but did not elucidate the underlying molecular mechanism. It has been recently reported that E7 from both HPV16 and HPV18 are sufficient to induce the expression of angiogenic factors (Smola-Hess et al, 2005; Bodily et al, 2011), and that high-risk HPVE7 can enhance tumour angiogenesis by inducing HIF- $1 \alpha$ protein accumulation and VEGF expression via activation of the ERK1/2 signalling pathway. We therefore hypothesised that RRM2, as a downstream target gene of HPVE7, might mediate HPVE7-induced HIF- $1 \alpha$ and VEGF expression via activation of the ERK1/2 signalling pathway. Indeed, we found that
RRM2 overexpression activated the ERK1/2 signalling pathway, and further showed that ERK1/2 activation was involved in RRM2induced HIF- $1 \alpha$ protein accumulation and VEGF expression in cervical cancer cells, and that these effects were abrogated by knockdown of RRM2 expression in cervical cancer cells. More importantly, our functional studies have shown that HPVE7 stimulated in vitro capillary tube formation and this effect was suppressed by RRM2 knockdown. In this study, we also investigated the mechanism by which RRM2 overexpression leads to the activation of the ERK1/2 pathway. Xue et al (2006) showed that RRM2 might induce the generation of ROS in vitro. ROS was implicated in the activation of the ERK1/2 pathway and can regulate HIF- $1 \alpha$ and VEGF expression. Our studies showed that RRM2 overexpression increased ROS production, whereas RRM2 knockdown decreased ROS production in cervical cancer cells. Furthermore, NAC, a ROS inhibitor, prevented the effect of RRM2 on activation of the ERK1/2 pathway and upregulation of VEGF expression. These findings suggest for the first time that RRM2 can induce ROS production to activate the ERK1/2 pathway, which then promotes VEGF expression in cervical cancer cells. However, more studies are required to provide further insights into the detailed molecular mechanisms by which RRM2 induces activation of ERK1/2 via ROS.

The RRM2 gene is located on chromosome 2p25, a region where no copy-number variations (CNVs) or other structural alterations were found in human cervical cancer samples (Policht et al, 2010). Therefore, the expression of RRM2 may not be influenced by $\mathrm{CNV}$ s or structural variants. Instead, HPVE7-induced overexpression may be a novel potential mechanism for upregulation of RRM2 expression in cervical cancer. Several reports showed that RRM2 was an independent prognostic factor and could predict poor survival of some cancer types (Morikawa et al, 2010). It is of interest to further investigate whether RRM2 could act as a biomarker for predicting the prognosis and survival of cervical cancer in the future. In conclusion, the results from this study demonstrate that high-risk HPVE7 upregulates expression of RRM2 at the transcriptional level; HPVE7 interacts with pRB and releases E2F, which binds to the RRM2 promoter and activates its transcription; RRM2 increases the level of intracellular ROS to promote HIF- $1 \alpha$ and VEGF expression via activating the ERK1/2 signalling pathway; increased VEGF expression results in increased tumour angiogenesis and promotes cervical carcinogenesis. The results provide a new perspective that RRM2 might be a therapeutic target for developing treatments for cervical cancer.

\section{ACKNOWLEDGEMENTS}

This work was supported by the Chinese National Natural Science Foundation Grant No. 81372803, No. 81172465, No. 30700984 and No. 81070080, the National Basic Research (973) Program grants 2012CB517801 and 2013CB0531101, a Hubei Province Natural Science Key Program (2008CDA047), a Key Academic Program Leader Award of Wuhan City (200951830560), and a grant from the State Key Laboratory of Freshwater Ecology and Biotechnology (2011FB16).

\section{REFERENCES}

Bodily JM, Mehta KP, Laimins LA (2011) Human papillomavirus E7 enhances hypoxia-inducible factor 1-mediated transcription by inhibiting binding of histone deacetylases. Cancer Res 71(3): 1187-1195.

Bosch FX, de SS (2007) The epidemiology of human papillomavirus infection and cervical cancer. Dis Markers 23: 213-227. 
Boyer SN, Wazer DE, Band V (1996) E7 protein of human papilloma virus-16 induces degradation of retinoblastoma protein through the ubiquitinproteasome pathway. Cancer Res 56: 4620-4624.

Branca M, Giorgi C, Santini D, Di Bonito L, Ciotti M, Benedetto A, Paba P, Costa S, Bonifacio D, Di Bonito P, Accardi L, Favalli C, Syrjänen K. HPVPathogen ISS Study Group (2006) Aberrant expression of VEGF-C is related to grade of cervical intraepithelial neoplasia (CIN) and high risk HPV, but does not predict virus clearance after treatment of CIN or prognosis of cervical cancer. J Clin Pathol 59: 40-47.

Drigotas M, Affolter A, Mann WJ, Brieger J (2013) Reactive oxygen species activation of MAPK pathway results in VEGF upregulation as an undesired irradiation response. J Oral Pathol Med 42(8): 612-619.

Duxbury MS, Whang EE (2007) RRM2 induces NF-kappaB-dependent MMP-9 activation and enhances cellular invasiveness. Biochem Biophys Res Commun 354: 190-196.

el-Deiry WS, Tokino T, Waldman T, Oliner JD, Velculescu VE, Burrell M, Hill DE, Healy E, Rees JL, Hamilton SR, Kinzler KW, Vogelstein B (1995) Topological control of p21WAF1/CIP1 expression in normal and neoplastic tissues. Cancer Res 55: 2910-2919.

Elford HL, Freese M, Passamani E, Morris HP (1970) Ribonucleotide reductase and cell proliferation. I.Variations of ribonucleotide reductase activity with tumor growth rate in a series of rat hepatomas. J Biol Chem 245: 5228-5233.

Fan H, Villegas C, Huang A, Wright JA (1998) The mammalian ribonucleotide reductase $\mathrm{R} 2$ component cooperates with a variety of oncogenes in mechanisms of cellular transformation. Cancer Res 58: 1650-1653.

Faoro V, Barbazza R, Bonin S, Brunetti D, Sulfaro S, Stanta G (2013) Detection of HPV E7 oncoviral protein in cervical lesions by a new antibody. Appl Immunohistochem Mol Morphol 21: 341-350.

Garner-Hamrick PA, Fostel JM, Chien WM, Banerjee NS, Chow LT, Broker TR, Fisher C (2004) Global effects of human papillomavirus type 18 E6/E7 in an organotypic keratinocyte culture system. J Virol 78: 9041-9050.

Gautam A, Li ZR, Bepler G (2003) RRM1-induced metastasis suppression through PTEN-regulated pathways. Oncogene 22: 2135-2142.

Gabellini C, Del Bufalo D, Zupi G (2006) Involvement of RB gene family in tumor angiogenesis. Oncogene 25: 5326-5332.

Holland D, Hoppe-Seyler K, Schuller B, Lohrey C, Maroldt J, Dürst M, HoppeSeyler F (2008) Activation of the enhancer of zeste homologue 2 gene by the human papillomavirus E7 oncoprotein. Cancer Res 68: 9964-9972.

Huh K, Zhou X, Hayakawa H, Cho JY, Libermann TA, Jin J, Harper JW, Munger K (2007) Human papillomavirus type 16 E7 oncoprotein associates with the cullin 2 ubiquitin ligase complex, which contributes to degradation of the retinoblastoma tumor suppressor. J Virol 81: 9737-9747.

Huh KW, DeMasi J, Ogawa H, Nakatani Y, Howley PM, Münger K (2005) Association of the human papillomavirus type $16 \mathrm{E} 7$ oncoprotein with the 600-kDa retinoblastoma protein-associated factor, p600. Proc Natl Acad Sci USA 102: 11492-11497.

Jemal A, Bray F, Center MM, Ferlay J, Ward E, Forman D (2011) Global cancer statistics. CA Cancer J Clin 61: 69-90.

Kuner R, Vogt M, Sultmann H, Buness A, Dymalla S, Bulkescher J, Fellmann M, Butz K, Poustka A, Hoppe-Seyler F (2007) Identification of cellular targets for the human papillomavirus E6 and E7 oncogenes by RNA interference and transcriptome analyses. J Mol Med 85: 1253-1262.

Katich SC, Zerfass-Thome K, Hoffmann I (2001) Regulation of the Cdc25A gene by the human papillomavirus Type 16 E7 oncogene. Oncogene 20: 543-55031.

Landt S, Mordelt K, Schwidde I, Barinoff J, Korlach S, Stöblen F, Lichtenegger W, Sehouli J, Kümmel S (2011) Prognostic significance of the angiogenic factors angiogenin, endoglin and endostatin in cervical cancer. Anticancer Res 31: 2651-2655.

Lee DJ, Kang SW (2013) Reactive oxygen species and tumor metastasis. Mol Cells 35: 93-98.

Liu H, Xiao J, Yang Y, Liu Y, Ma R, Li Y, Deng F, Zhang Y (2011) COX-2 expression is correlated with VEGF-C, lymphangiogenesis and lymph node metastasis in human cervical cancer. Microvasc Res 82: 131-140.

Liu X, Zhang H, Lai L, Wang X, Loera S, Xue L, He H, Zhang K, Hu S, Huang Y, Nelson RA, Zhou B, Zhou L, Chu P, Zhang S, Zheng S, Yen Y (2013) Ribonucleotide reductase small subunit M2 serves as a prognostic biomarker and predicts poor survival of colorectal cancers. Clin Sci 124: 567-578.

Meng D, Mei A, Liu J, Kang X, Shi X, Qian R, Chen S (2012) NADPH oxidase 4 mediates insulin-stimulated HIF- $1 \alpha$ and VEGF expression, and angiogenesis in vitro. PLoS One 7: e48393.

Morikawa T, Maeda D, Kume H, Homma Y, Fukayama M (2010) Ribonucleotide reductase M2 subunit is a novel diagnostic marker and a potential therapeutic target in bladder cancer. Histopathology 57: 885-892.
Nordlund P, Reichard P (2006) Ribonucleotide reductases. Annu Rev Biochem 75: 681-706.

Policht FA, Song M, Sitailo S, O’Hare A, Ashfaq R, Muller CY, Morrison LE, King W, Sokolova IA (2010) Analysis of genetic copy number changes in cervical disease progression. BMC Cancer 10: 432.

Ressler S, Scheiden R, Dreier K, Laich A, Müller-Holzner E, Pircher H, Morandell D, Stein I, Viertler HP, Santer FR, Widschwendter A, Even J, Jansen-Dürr P, Capesius C, Zwerschke W (2007) High-risk human papillomavirus E7 oncoprotein detection in cervical squamous cell carcinoma. Clin Cancer Res 13: 7067-7072.

Rosty C, Sheffer M, Tsafrir D, Stransky N, Tsafrir I, Peter M, De Crémoux P, de La Rochefordière A, Salmon R, Dorval T, Thiery JP, Couturier J, Radvanyi F, Domany E, Sastre-Garau X (2005) Identification of a proliferation gene cluster associated with HPV E6/E7 expression level and viral DNA load in invasive cervical carcinoma. Oncogene 24: 7094-7104.

Smola-Hess S, Pahne J, Mauch C, Zigrino P, Smola H, Pfister HJ (2005) Expression of membrane type 1 matrix metalloproteinase in papillomavirus-positive cells: role of the human papillomavirus (HPV) 16 and HPV8 E7 gene products. J Gen Virol 86(Pt 5): 1291-1296.

Tang S, Tao M, McCoy Jr JP, Zheng ZM (2006) The E7 oncoprotein is translated from spliced E6 ${ }^{\star}$ I transcripts in high-risk human papillomavirus type 16- or type 18-positive cervical cancer cell lines via translation reinitiation. J Virol 80: 4249-4263.

Tang X, Zhang Q, Nishitani J, Brown J, Shi S, Le AD (2007) Overexpression of human papillomavirus type 16 oncoproteins enhances hypoxia-inducible factor 1 alpha protein accumulation and vascular endothelial growth factor expression in human cervical carcinoma cells. Clin Cancer Res 13: 2568-2576.

Thierry F, Benotmane MA, Demeret C, Mori M, Teissier S, Desaintes C (2004) A genomic approach reveals a novel mitotic pathway in papillomavirus carcinogenesis. Cancer Res 64: 895-903.

Tian J, Yan J, Wang W, Zhong N, Tian L, Sun J, Min Z, Ma J, Lu S (2012) T-2 toxin enhances catabolic activity of hypertrophic chondrocytes through ROS-NF-אB-HIF-2 $\alpha$ pathway. Toxicol In Vitro 26: 1106-1113.

Tian XL, Kadaba R, You SA, Liu M, Timur AA, Yang L, Chen Q, Szafranski P, Rao S, Wu L, Housman DE, DiCorleto PE, Driscoll DJ, Borrow J, Wang Q (2004) Identification of an angiogenic factor that when mutated causes susceptibility to Klippel-Trenaunay syndrome. Nature 42: 640-645.

Tsai HH, Lee WR, Wang PH, Cheng KT, Chen YC, Shen SC (2013) Propionibacterium acnes-induced iNOS and COX-2 protein expression via ROS-dependent NF- $\mathrm{\kappa B}$ and AP-1 activation in macrophages. J Dermatol Sci 69: 122-131.

Wang F, Xu CQ, He Q, Cai JP, Li XC, Wang D, Xiong X, Liao YH, Zeng QT, Yang YZ, Cheng X, Li C, Yang R, Wang CC, Wu G, Lu QL, Bai Y, Huang YF, Yin D, Yang Q, Wang XJ, Dai DP, Zhang RF, Wan J, Ren JH, Li SS, Zhao YY, Fu FF, Huang Y, Li QX, Shi SW, Lin N, Pan ZW, Li Y, Yu B, Wu YX, Ke YH, Lei J, Wang N, Luo CY, Ji LY, Gao LJ, Li L, Liu H, Huang EW, Cui J, Jia N, Ren X, Li H, Ke T, Zhang XQ, Liu JY, Liu MG, Xia H, Yang B, Shi LS, Xia YL, Tu X, Wang QK (2011) Genome-wide association identifies a susceptibility locus for coronary artery disease in the Chinese Han population. Nat Genet 43: 345-349.

Werness BA, Levine AJ, Howley PM (1990) Association of human papillomavirus types 16 and 18 E6 proteins with p53. Science 248: 76-79.

Xia C, Meng Q, Liu LZ, Rojanasakul Y, Wang XR, Jiang BH (2007) Reactive oxygen species regulate angiogenesis and tumor growth through vascular endothelial growth factor. Cancer Res 67: 10823-10830.

Xue L, Zhou B, Liu X, Wang T, Shih J, Qi C, Heung Y, Yen Y (2006) Structurally dependent redox property of ribonucleotide reductase subunit p53R2. Cancer Res 66: 1900-1905.

Zhang K, Hu S, Wu J, Chen L, Lu J, Wang X, Liu X, Zhou B, Yen Y (2009) Overexpression of RRM2 decreases thrombspondin-1 and increases VEGF production in human cancer cells in vitro and in vivo: implication of RRM2 in angiogenesis. Mol Cancer 8: 11-22.

Zhou B, Ma R, Si W, Li S, Xu Y, Tu X, Wang Q (2013) MicroRNA-503 targets FGF2 and VEGFA and inhibits tumor angiogenesis and growth. Cancer Lett 333: 159-169.

Zhou BS, Tsai P, Ker R, Tsai J, Ho R, Yu J, Shih J, Yen Y (1998) Overexpression of transfected human ribonucleotide reductase M2 subunit in human cancer cells enhances their invasive potential. Clin Exp Metastasis 16: 43-49.

This work is published under the standard license to publish agreement. After 12 months the work will become freely available and the license terms will switch to a Creative Commons AttributionNonCommercial-Share Alike 3.0 Unported License. 